\title{
A Simple Self-Rating Assessment Method of Residual Work Capability for Occupational Permanent Disabilities
}

\author{
Yu-Chen Chang, MD, ${ }^{1}$ Mei-Jin ChenSea, ScD, ${ }^{2}$ Yuh Jang, otR, MHE, ${ }^{3}$ \\ and Jung-Der Wang, MD, SCD ${ }^{1,4}$
}

\begin{abstract}
Background To explore the validity, reliability, and determinants of a simple selfrating assessment method of residual work capability (RWC) after occupational permanent disabilities.

Methods Five hundred and thirty-nine compensated permanent disability workers answered three consecutive visual analogue questionnaires wherein they self-rated their residual work capability in terms of speed (RWCS), quality (RWCQ) and a combination of speed and quality (RWCC). At two major hospitals in Taiwan, 169 of these subjects were evaluated with physical capacity assessment (PCA), cognition and sensation assessment (CSA), the work ability index (WAI), and the 12-item Chinese health questionnaire (CHQ-12).

Results High test-retest reliability (Pearson's correlation coefficient 0.77) and satisfactory concurrent validity were shown for RWCS and RWCC. All PCA, CSA and WAI showed significant correlation with RWCs, while CHQ-12 displayed borderline correlation. Employment status after injury and status of the victim's salary as the main source of income for his/her family before injury, were the major determinants of RWCs, in addition to the scales of PCA, CSA and WAI.

Conclusions The RWCC resulting from the self-rating method may be used as a simple assessment of a victim's residual work capability after occupational permanent disabilities. Am. J. Ind. Med. 38:539-547, 2000. ๑ 2000 Wiley-Liss, Inc.
\end{abstract}

KEY WORDS: work capability; occupational injury; occupational rehabilitation

\footnotetext{
${ }^{1}$ Center for Research of Environmental and Occupational Diseases, Institute of Occupational Medicine and Industrial Hygiene, National Taiwan University College of Public Health, Taipei, Taiwan

${ }^{2}$ School of Occupational Therapy, College of Medicine, National Cheng Kung University, Tainan, Taiwan

${ }^{3}$ School of Occupational Therapy, College of Medicine, National Taiwan University, Taipei, Taiwan

${ }^{4}$ Department of Internal Medicine, National Taiwan University Hospital

Abbreviations: RWCS, Residual work capability (in percentage points) of workproductivity in terms of speed compared with that before the injury by respondent's selfrating on a visual analogue rating scale; RWCQ, Residual work capability (in percentage points) of work productivity in terms of quality compared with that before the injury by respondent's self-rating on a visual analogue rating scale; RWCC, Residual work capability (in percentage points) of work productivity in terms of a combination of speed and quality
}

compared with that before the injury by respondent's self-rating on a visual analogue rating scale; PCA: Physical capacity assessment of subjects by occupational therapists; WAI Finnish work ability index, CSA: Cognition and sensation assessment, CHQ-12; Chinese health questionnaire, 12-items version, ERWC; Estimation of residual work capability from workmen's compensation scheme as enforced by the Taiwan Labor Law (1,800 lost workdays, assuming $100 \%$ loss of work capability, serves as the baseline for ERWC)

Contract grant sponsor: The Council of Labor Affairs, ExecutiveYuan, ROC; Contract grant number: IOSH-84-M203

${ }^{*}$ Correspondence to: Dr. Jung-Der Wang, Center for Research of Environmental and Occupational Diseases, Institute of Occupational Medicine and Industrial Hygiene, National Taiwan University College of Public Health, No. 1, Section 1, Jen-Ai Road, Taipei, Taiwan, ROC. 10016. E-mail: jdwang@ ha.mc.ntu.edu.tw

Accepted 7 January 2000 


\section{INTRODUCTION}

Measuring the impact of severe occupational injuries solely with mortality rates is an inadequate indicator. In the past decade in Taiwan, the average ratio of deaths-topermanent disabilities from occupational injury was about 1:3.5 [Bureau of Labor Insurance for Taiwan-Fukein Area, 1984-1994]. Presently, due to the complex combinations of various impairments of a victim's physical, mental and/or other health conditions after permanent injury, there is still a lack of consensus on a single index to represent the severity of permanent disability [Tuomi et al., 1994]. Although the disabling severity rate has been widely adopted as an indicator for quantifying work disability from occupational injury [American National Standards Institute, 1967], the arbitrary weighting factors applied to conditions of permanent disability indicate the need for further studies to develop alternative quantification methods [Chang and Wang, 1995]. Furthermore, the indices of residual work capability from different occupational permanent disabilities would assist in estimating the cost of occupational injuries to human resources and accounting [Gojer and Johanson, 1991].

Determining a healthy worker's real work capability is a difficult task and probably cannot be measured by a single figure [Tuomi et al., 1994; Chang and Wang, 1995]. Several authors have pointed out that work capability usually involves activities related to personal care, mobility, sensory perception, communication, recreation, socializing, and intimacy, according to the definition of work capability [Gulick, 1992]. It is also related to mental and intellectual status, psychological distress, individual work environment, various industrial and occupational requirements, and even personal demographic characteristics [Abdel-Moty et al., 1993; Velozo, 1993]. Methods that measure certain dimensions of work capability, such as functional capacity [AbdelMoty et al., 1993], physical performance components, [Mathiowetz, 1993] or physical work performance [Lechner et al., 1994], are generally considered to be insufficient measurements of real work capability [Velozo, 1993; Tuomi et al., 1994].

The work ability index (WAI), developed by the Finnish Institute of Occupational Health [Tuomi et al., 1994], may be the only instrument with high validity which can evaluate the subjective work ability of a worker with an integrated number during longitudinal follow-up. However, as injuries often result from unexpected accidents, the WAI may not be directly used for the assessment of residual work capability unless one has baseline data. It requires at least two measurements of the individual WAI before and after the injury event. In addition, we must assume that WAI is a linear scale to obtain the residual percentage of work ability.

To confront the above difficulties, we chose to examine the concept of self-comparison and bypass multi-dimen- sional measurements by directly asking subjects to self-rate his/her own RWC on speed and quality. The aim of this study was to introduce and to validate a simple subjective rating method for determining RWC after permanent occupational disability. To validate the new method, we simultaneously applied the measurements of WAI (an overall index), physical capacity assessment (PCA), and cognition and sensation assessment (CSA) for comparison.

\section{MATERIALS AND METHODS}

\section{Subjects}

There were 14,084 compensated cases of traumatic occupational permanent disability $(11,050$ men and 3,034 women) in Taiwan from January 1990 through December 1992, as shown in Table I. All disabilities were diagnosed and classified by certificate based on 160 well-defined conditions listed in the Workmen's Compensation Scheme of Taiwan. These were then categorized into 15 severity grades of permanent disability for the convenience of compensation payment [Regulation of Labor Insurance, 1992]. The first grade of permanent disability corresponds to the permanent total disability of the American National Standards Institute (ANSI) system, and the other 14 grades of permanent disability correspond to ANSI's permanent partial disability. Stratified random sampling was performed according to gender, the calendar year in which occupational injuries occurred and the 15 severity grades of permanent disability. A total of 2,145 (1,299 men and 846 women) permanent disability workers from nine industrial sectors were drawn from the archives of the compensation registry of the Taiwan Labor Insurance Bureau from January 1990 through December 1992.

\section{Instrumentation}

\section{A questionnaire on residual work capability}

A questionnaire was constructed and mailed to the selected subjects about their residual work capability after occupational injury. By dividing work capability into two major domains, the speed of completing a task and the quality of execution, the questionnaire was designed to draw a percentage profile of the RWC for injured and compensated workers. The questions, as literally translated from Chinese, are as follows: First question, "Please mark the appropriate percentage on the following rating scales which represents your residual work capability relating to productivity in terms of your current speed compared with that before the injury" (RWCS). The second question: "Please mark the appropriate percentage on the following rating scales which represents your residual work capability 
relating to productivity in terms of the quality of your work compared with that before the injury" (RWCQ). The third question: "Please mark the appropriate percentage on the following rating scales which represents your residual work capability relating to productivity in terms of a combination of both speed and quality compared with that before the injury" (RWCC). Each question was presented with a visual analogue rating scale in the form of a thermometer, where $100 \%$ represented "no work capability loss compared with that before the injury" and $0 \%$ represented "total loss of work capability" (such as quadriplegia or complete blindness in both eyes). The three questions were arranged in consecutive sequences for RWCS, RWCQ and RWCC. Additionally, it was asked whether the salary of the injured worker was the main source income of his/her family before the injury (IS), and whether the victim returned to work after the injury (to either the same or a different job) (RTW). (The full text of the questionnaires is available upon request from the corresponding author.)

\section{The physical capacity assessment (PCA)}

The physical capacity assessment was developed for the field of occupational therapy based on the physical capacity requirements of the Dictionary of Occupational Titles, U.S. Department of Labor [Smith, 1988]. PCA involves the following six domains of measurements: weight capacity assessment with four items, flexibility assessment with three items, ambulatory skills with three items, static positioning assessment with two items, hand grip and finger pinch of both hands with one item each, and handling capacity with two items each. Hand strength was measured by gripping a dynamometer, and finger pinch strength was measured with a pinch gauge. The recommendations for standardized positioning and established procedures and instructions were followed for each test of strength [Mathiowetz, 1990]. These tests have been reported to achieve high inter-rater and test-retest reliability [Mathiowetz et al., 1984]. Handling capacity was assessed by both the Purdue pegboard [Tiffin and Asher, 1948] and the Minnesota Rate of Manipulation Test [Parry, 1972], according to standard procedures. This scoring system yielded six raw scores for each physical domain of capability, which were then summed up to obtain a total score. The ranges of scores were as follows: $0-21$ for weight capacity, $0-16$ for flexibility, $0-16$ for ambulatory skills, $0-6$ for static positioning, $0-12$ for grip and pinch, and $0-21$ for handling capability.

\section{The cognition and sensation assessment (CSA)}

This assessment included two dimensions, cognition and sensation. These were extracted and modified from the Position Analysis Questionnaire [McCormick et al., 1989].
Cognition measurement involves the ability to understand a command, to express an idea, and to recall and perform a command, which is scored from 4 (worst) to 16 (best). Sensation measurement involves visual, auditory, and tactile functions and is scored from the worst condition to the best condition (3-12). The results of these two assessments were all recorded by occupational therapists during the tests for physical capacity.

\section{The work ability index (WAI)}

The WAI questionnaire developed by the Finnish Institute of Occupational Health is composed of seven items with 10 questions inquiring about work ability [Tuomi et al., 1991]. The conceptual definition of WAI comes from the question: "How good is the worker at present, in the near future, and how able is he or she to do his or her work with respect to work demands, health and mental resources?" The Chinese version that was used is a direct translation from the English version with minimal modification of culturally relevant terms. The WAI is derived as the sum of the seven items. The range of the WAI is 7-49. A low score of WAI represents poorer work ability, while a high score represents the converse. Previously, the WAI was demonstrated to be a good predictor of the possibility of workers receiving disability pension during a 5-year follow-up study of 2,693 workers [Tuomi et al., 1994].

\section{The 12-item Chinese health questionnaire (CHQ-12)}

This questionnaire was developed from a Chinese translation of the General Health Questionnaire [Goldberg, 1972] with the modification and addition of several specially designed, culturally relevant items [Cheng and Williams, 1986] to improve physicians' identification of minor psychiatric disorders in Chinese patients. The four categories of questions in the CHQ-12 cover the assessment of (1) anxiety and depression, (2) the respondent's concern over sleep disturbance, (3) somatic symptoms/concern, (4) interpersonal difficulties [Cheng and Williams, 1986; Chong and Wilkinson, 1989; Cheng et al., 1990], with the validation of sensitivity rate of $78 \%$ and specificity rate of $77 \%$ [Chong and Wilkinson, 1989]. We used the CHQ-12 as a screening questionnaire to detect the emotional distress of the injured workers. A CHQ-12 score ranges from 0 to 36 . A low score represents less emotional distress, and a high score represents more emotional distress.

\section{The estimation of residual work capability (ERWC)}

The ERWC is a product of the current Workmen's Compensation Scheme enforced in Taiwan [Regulation of 
Labor Insurance, 1992], in which the grade of permanent disability is based on preexisting tables of lost workdays. There are 15 grades of permanent disabilities, and the higher the grade, the less severe the disability. For example, the first grade of permanent total disability is a complete loss of work capability (i.e. equal to $100 \%$ loss), and the workmen's compensation system of Taiwan pays up to 1800 lost workdays as a baseline. We divided the corresponding number of paid workdays lost by the same compensation system by 1800 workdays to obtain the percentage of work capability lost permanently. This was then subtracted from $100 \%$ to get the ERWC. For example, occupational permanent disability resulting in above the knee amputation of one extremity was paid up to 960 workdays for compensation, which was categorized as the fifth grade of permanent disability. The ERWC is calculated to be $100 \%-(960 /$ $1800)=46.7 \%$. All the other 14 grades of permanent disability were calculated in the same way to obtain the ERWCs [Chang and Wang, 1995].

\section{Statistical Analysis}

The SAS program (version 6.08) was used for statistical analysis [SAS, 1990]. The test-retest reliability was evaluated by the Pearson correlation coefficient for consistency between responses, ( 3 months apart) to the same questionnaires on residual work capability after permanent disability. To determine concurrent criterionrelated validity, the Pearson correlation coefficient was also calculated to show the correlation between the RWC scores and the scores from PCA, CSA, WAI and CHQ-12. Multiple regression analysis was used to fit the best-predicted models for the RWCs as dependent variables, and the backward selection method was used to find out the major determinants of the RWCs. In addition to PCA, CSA, WAI, and CHQ-12, we also included the demographic variables (age, gender, marital status, education) in the multiple regression analysis to fit the models.

\section{Procedures}

Subjects were asked and provided with instructions to fill out the pre-tested self-administered questionnaire inquiring about their residual work capability after permanent disability. Efforts to recruit non-responding subjects for the questionnaire, by re-mailing and telephone follow up, were repeated twice at 1-month intervals after mailing the first questionnaire in November 1994. Among the respondents who returned the questionnaire, we invited everyone to participate in a further physical examination to be conducted in a hospital. Similarly, two more rounds of telephone and mail invitations were sent, at 1-month intervals.

Subjects were asked to answer several demographic questions and provide information on their residual work capability due to permanently disabling injuries. The total time needed to fill out the mailed questionnaire was about 15 min according to pretests. The National Taiwan University Hospital located in Taipei, northern Taiwan, and the Tainan Municipal Hospital located in southern Taiwan served as the two sites for physical examination. All participants who attended the physical examination were required to answer a brief version of the mailed questionnaire again, in addition to completing the tests and questionnaires for PCA, CSA, WAI and CHQ-12. The total time to complete the physical examination required about $1-1.5 \mathrm{~h}$ for each participant on average. Physical examinations were performed by doctors, while the physical capacity assessment and cognition and sensation assessments were performed by occupational therapists. Laboratory tests included serum biochemistry, complete blood count, and urine tests.

\section{RESULTS}

Table I summarizes the distribution of different grades of permanent disability for frequencies of random samples,

TABLE I. Total Number of Cases with Permanent Disability (T), Frequencies of Random Samples (RS), Respondents of Questionnaire (Q), and Participants of Physical Examination (PE) Stratified by Grade of Permanent Disability from January 1990 through December 1992, Taiwan

Grade of permanent disability*

\begin{tabular}{lrrrrrrrrrrrrrrrr}
\hline & $\mathbf{1}$ & $\mathbf{2}$ & $\mathbf{3}$ & $\mathbf{4}$ & $\mathbf{5}$ & $\mathbf{6}$ & $\mathbf{7}$ & $\mathbf{8}$ & $\mathbf{9}$ & $\mathbf{1 0}$ & $\mathbf{1 1}$ & $\mathbf{1 2}$ & $\mathbf{1 3}$ & $\mathbf{1 4}$ & $\mathbf{1 5}$ & Total \\
\hline $\mathrm{T}$ & 88 & 156 & 54 & 11 & 145 & 279 & 306 & 771 & 891 & 942 & 2,223 & 3,565 & 2,335 & 1,172 & 1,146 & 14,084 \\
$\mathrm{RS}$ & 76 & 140 & 47 & 8 & 136 & 133 & 113 & 184 & 185 & 187 & 190 & 187 & 188 & 183 & 188 & 2,145 \\
$\mathrm{Q}$ & 22 & 46 & 17 & 2 & 43 & 38 & 31 & 52 & 50 & 38 & 44 & 37 & 42 & 38 & 39 & 539 \\
$\mathrm{PE}$ & 3 & 10 & 3 & - & 14 & 10 & 8 & 14 & 15 & 19 & 11 & 19 & 16 & 13 & 14 & 169 \\
\hline
\end{tabular}

*The higher the grade, the less severe the permanent disability. 
respondents to the mailed questionnaire, and participants of the physical examination held at the two hospitals. There were a total of $539(25.1 \%)$ respondents to the mailed questionnaire after two more cycles of re-mailing, 1 month apart. One hundred and sixty-nine subjects out of the 539 respondents $(31.2 \%)$ participated in the physical examination at the two hospitals 2 months later. Severe disabilities included grades 1-5, e.g., mental impairment, quadriplegia, loss of both hands, amputation at the elbow or above of at least one upper extremity, amputation at the knee joint or above of at least one lower extremity, etc. In general, every year, there were less than 50 cases of each grade of severe disability. Very few cases had been categorized as grade 4, possibly due to difficulty in classification and/or because few suitable conditions match grade 4 criteria, e.g., obvious motion impairment of both upper extremities or both lower extremities. The duration since onset of occupational injury to date of answering the questionnaire, ranged from 2.0 to 4.9 years $($ mean $=3.4, \mathrm{SD}=0.9) .539$ injured workers $(330$ men, 209 women, mean age 42.2, SD 12.5) responded to the

TABLE II. Comparisons of Demographic Characteristics Between Responding Workers Who Came and did not Come in for Further Physical Examination

\begin{tabular}{|c|c|c|c|c|}
\hline & & \multicolumn{2}{|c|}{ Physical examination } & \multirow[b]{2}{*}{$P$ value* } \\
\hline & & Yes & No & \\
\hline No. of workers & & 169 & 370 & \\
\hline \multirow[t]{2}{*}{ Age } & Mean (S.D.) & $43.1(11.9)$ & $41.8(12.8)$ & 0.27 \\
\hline & Range & $22-74$ & $19-68$ & \\
\hline Gender & Male/Female & $100 / 69$ & $230 / 140$ & 0.51 \\
\hline \multirow[t]{5}{*}{ Education } & Illiteracy & 20 & 48 & 0.90 \\
\hline & Primary school & 54 & 126 & \\
\hline & Junior high & 41 & 75 & \\
\hline & Senior high & 42 & 87 & \\
\hline & College/above & 11 & 26 & \\
\hline \multirow[t]{5}{*}{ Marital status } & Single & 25 & 69 & 0.64 \\
\hline & Married & 134 & 265 & \\
\hline & Loss of spouse & 6 & 17 & \\
\hline & Divorced & 4 & 6 & \\
\hline & Remarried & 0 & 2 & \\
\hline \multirow[t]{2}{*}{ Current employment status } & Employed & 46 & 87 & 0.78 \\
\hline & Unemployed & 106 & 213 & \\
\hline \multirow[t]{15}{*}{ Grades of permanent disability ${ }^{a}$} & 1 & 3 & 19 & 0.24 \\
\hline & 2 & 13 & 33 & \\
\hline & 3 & 8 & 9 & \\
\hline & 4 & 1 & 1 & \\
\hline & 5 & 10 & 33 & \\
\hline & 6 & 10 & 28 & \\
\hline & 7 & 7 & 24 & \\
\hline & 8 & 15 & 37 & \\
\hline & 9 & 17 & 33 & \\
\hline & 10 & 12 & 26 & \\
\hline & 11 & 21 & 23 & \\
\hline & 12 & 15 & 22 & \\
\hline & 13 & 16 & 26 & \\
\hline & 14 & 11 & 27 & \\
\hline & 15 & 10 & 29 & \\
\hline
\end{tabular}


TABLE III. Correlation Matrix of the RWC and Various Measurements of Workers' Physical and Psychological Status

\begin{tabular}{|c|c|c|c|c|c|c|c|}
\hline & RWCQ & RWCC & PCA & WAI & CSA & CHQ-12 & ERWC \\
\hline \multirow[t]{3}{*}{ RWCS } & 0.73 & 0.94 & 0.67 & 0.84 & 0.40 & -0.16 & 0.73 \\
\hline & 0.00 & 0.00 & 0.00 & 0.00 & 0.00 & 0.04 & 0.00 \\
\hline & 163 & 163 & 153 & 162 & 149 & 162 & 163 \\
\hline \multirow[t]{3}{*}{ RWCQ } & & 0.81 & 0.52 & 0.68 & 0.40 & -0.14 & 0.58 \\
\hline & & 0.00 & 0.00 & 0.00 & 0.00 & 0.08 & 0.00 \\
\hline & & 163 & 153 & 162 & 149 & 162 & 163 \\
\hline \multirow[t]{3}{*}{ RWCC } & & & 0.69 & 0.86 & 0.38 & -0.14 & 0.74 \\
\hline & & & 0.00 & 0.00 & 0.00 & 0.07 & 0.00 \\
\hline & & & 153 & 162 & 149 & 162 & 163 \\
\hline \multirow[t]{3}{*}{ PCA } & & & & 0.65 & 0.29 & -0.03 & 0.76 \\
\hline & & & & 0.00 & 0.00 & 0.73 & 0.00 \\
\hline & & & & 156 & 149 & 156 & 157 \\
\hline \multirow[t]{3}{*}{ WAI } & & & & & 0.39 & -0.23 & 0.66 \\
\hline & & & & & 0.00 & 0.00 & 0.00 \\
\hline & & & & & 152 & 167 & 167 \\
\hline \multirow[t]{3}{*}{ CSA } & & & & & & -0.22 & 0.27 \\
\hline & & & & & & 0.01 & 0.00 \\
\hline & & & & & & 152 & 152 \\
\hline \multirow[t]{3}{*}{ CHQ-12 } & & & & & & & 0.04 \\
\hline & & & & & & & 0.61 \\
\hline & & & & & & & 167 \\
\hline
\end{tabular}

Please refer to the abbreviation list in the text.

Top to bottom in each unit: Pearson's correlation coefficient, $P$ value, sample size (the $P$ value 0.00 means $<0.0001$ ).

questionnaire and 169 (100 men, 69 women, mean age 43.1, SD 11.9) agreed to participate in the physical examination. A comparison of several variables between the groups who took and those who did not take the physical examination showed no significant differences in terms of demographic items, employment status after injury and distribution of permanent disability grade (Table II).

The test-retest reliability (conducted 3 months later) for a respondent's rating of his/her residual work capability, in terms of speed (RWCS), quality (RWCQ), and combination of speed and quality (RWCC), were found to be 0.77 , 0.58 and 0.77 (all $P<0.0001$ ), respectively. The test-retest reliability of the RWCS, RECQ and RWCC questions was high. The internal consistency reliability of the RWCS, RWCQ and RWCC questions with each other was high (Table III). The RWCC was highly correlated with RWCS $(r=0.94)$ and RWCQ $(r=0.81)$; however, RWCS showed slightly lower correlation with RWCQ $(r=0.73)$, but all $P<0.0001$.

To determine the validity of the questionnaire, we used several indicators to calculate the correlation coefficients of concurrent criterion-related validity. Table III shows the correlation matrix between these various indicators. RWCS and RWCC showed higher correlation with PCA $(r=0.67$; $r=0.69)$ than RWCQ with PCA (0.52). High correlation was also noticed between WAI, RWCS, and RWCC ( 0.84 and 0.86 , respectively), and WAI showed relatively low correlation with RWCQ (0.68) and PCA (0.65). Between CSA and RWCs, the correlation was almost of the same level $(r=0.4)$, and there was a similar magnitude of correlation between CSA and WAI. The correlation of the RWCS, RWCQ, and RWCC questions with the WAI, PCA, and CSA was high (all $P<0.0001$ ). The CHQ-12 displayed mildly significant negative correlation with RWCs, WAI, and CSA, and no correlation with PCA. ERWC showed highly significant correlation coefficients with RWCS, RWCC, and PCA, moderate correlation with RWCQ, WAI, and no correlation with CHQ-12.

Using multiple regression analysis, the best models for RWCs as dependent variables were fitted to find the significant determinants (Table IV). High $R^{2}(0.77)$ was found in the RWCC as a dependent variable. WAI, PCA, and the variable of whether a victim's salary was the main source of income for his/her family before injury (variable IS) were found to be significant determinants in the model. Gender, age, education level, marital status, and employment status after injury (variable RTW) did not appear in the models. A similar finding was also found in the model of RWCS. However, in the model of RWCQ, CSA instead of PCA appeared in the model while the same variables of WAI 
TABLE IV. Determinants for the Self-Rating Method of Residual Work Capability

\begin{tabular}{|c|c|c|c|c|c|c|}
\hline \multirow{2}{*}{$\begin{array}{l}\text { Dependent } \\
\text { variable }\end{array}$} & \multirow{2}{*}{$\begin{array}{l}\text { Independent } \\
\text { variables }\end{array}$} & \multirow{2}{*}{$\begin{array}{l}\text { Parameter } \\
\text { estimate }\end{array}$} & \multirow{2}{*}{$\begin{array}{c}\text { Standard } \\
\text { error }\end{array}$} & \multirow[b]{2}{*}{$P$ value } & \multicolumn{2}{|c|}{ Partial } \\
\hline & & & & & $R^{2}$ & $\boldsymbol{R}^{2}$ \\
\hline \multicolumn{7}{|c|}{ Models fitted with independent variables including the WAI } \\
\hline \multirow[t]{4}{*}{ RWCS } & Intercept & -26.40 & 5.66 & 0.00 & & 0.73 \\
\hline & WAI & 2.00 & 0.19 & 0.00 & 0.47 & \\
\hline & PCA & 0.31 & 0.09 & 0.00 & 0.09 & \\
\hline & IS & 6.88 & 2.83 & 0.02 & 0.05 & \\
\hline \multirow[t]{4}{*}{ RWCQ } & Intercept & -83.97 & 36.67 & 0.02 & & 0.49 \\
\hline & WAI & 1.91 & 0.24 & 0.00 & 0.34 & \\
\hline & CSA & 3.29 & 1.35 & 0.02 & 0.05 & \\
\hline & IS & 7.19 & 4.26 & 0.09 & 0.02 & \\
\hline \multirow[t]{4}{*}{ RWCC } & Intercept & -30.83 & 5.36 & 0.00 & & 0.77 \\
\hline & WAl & 2.14 & 0.18 & 0.00 & 0.52 & \\
\hline & PCA & 0.31 & 0.09 & 0.00 & 0.10 & \\
\hline & IS & 6.91 & 2.68 & 0.01 & 0.05 & \\
\hline \multicolumn{7}{|c|}{ Models fitted with independent variables excluding the WAI } \\
\hline \multirow[t]{5}{*}{ RWCS } & Intercept & -96.38 & 28.81 & 0.00 & & 0.62 \\
\hline & PCA & 0.60 & 0.10 & 0.00 & 0.23 & \\
\hline & RTW & 21.18 & 4.29 & 0.00 & 0.17 & \\
\hline & IS & 8.50 & 3.39 & 0.01 & 0.05 & \\
\hline & CSA & 2.50 & 1.07 & 0.02 & 0.04 & \\
\hline \multirow[t]{5}{*}{ RWCQ } & Intercept & -111.19 & 38.61 & 0.01 & & 0.43 \\
\hline & PCA & 0.32 & 0.14 & 0.02 & 0.04 & \\
\hline & RTW & 23.38 & 5.75 & 0.00 & 0.12 & \\
\hline & IS & 8.67 & 4.54 & 0.06 & 0.03 & \\
\hline & CSA & 4.00 & 1.43 & 0.01 & 0.06 & \\
\hline \multirow[t]{5}{*}{ RWCC } & Intercept & -87.94 & 28.91 & 0.00 & & 0.63 \\
\hline & PCA & 0.63 & 0.10 & 0.00 & 0.25 & \\
\hline & RTW & 22.96 & 4.31 & 0.00 & 0.19 & \\
\hline & IS & 8.87 & 3.40 & 0.01 & 0.05 & \\
\hline & CSA & 2.00 & 1.07 & 0.07 & 0.03 & \\
\hline
\end{tabular}

IS: Whether the victim's salary was the main source of his/her family before injury $(1=y e s, 0=n 0)$.

RTW: Whether the victim returned to work after injury $(1=y e s, 0=$ no).

For other abbreviations, please refer to the abbreviation list in the text.

The demographic variables (age, gender, marital status, and education) were considered in these analyses but were not significant. Only significant predictors are shown here.

and IS were retained. Although CSA and CHQ-12 were partially correlated with all RWCs, they were replaced by WAI in the best-fitted model. The partial $R^{2}$ of each independent variable are also displayed in Table IV. The three models of RWCs, fitted with independent variables including WAI, showed a high partial $R^{2}$ for WAI in each model. Since WAI is a combined indicator including several aspects of measurement, it is worthwhile to try to fit the models of the same dependent variables and the same independent variables, with the exception of WAI. In the models fitted with the mentioned possible determinants excluding WAI, we found that in addition to the PCA and IS, the CSA and RTW entered the models with an $R^{2}$ slightly less than that of the models, including WAI with all RWCs as dependent variables. The partial $R^{2}$ of PCA and RTW were larger than the partial $R^{2}$ of IS and CSA with the dependent variables of RWCS and RWCC. However, the partial $R^{2}$ of RTW in the RWCQ model was larger in other models. If we took the WAI as a dependent variable and the RWCs as independent variables, the fitted results showed that each RWC had a very high sum of partial $R^{2}$ in each model fitted ( $\geq 0.93)$, as shown in Table V. 
TABLE V. Partial $R^{2}$ Between WAl and the Self-Rating Method of RWC

\begin{tabular}{llccccr}
$\begin{array}{l}\text { Dependent } \\
\text { variable }\end{array}$ & $\begin{array}{c}\text { Independent } \\
\text { variables }\end{array}$ & $\begin{array}{c}\text { Parameter } \\
\text { estimate }\end{array}$ & $\begin{array}{c}\text { Standard } \\
\text { error }\end{array}$ & $\boldsymbol{P}$ value & $\boldsymbol{R}^{\mathbf{2}}$ & $\boldsymbol{R}^{\mathbf{2}}$ \\
\hline WAI & Intercept & 12.13 & 0.92 & 0.00 & & 0.75 \\
& RWCS & 0.10 & 0.04 & 0.01 & 0.04 & \\
WAI & RWCC & 0.18 & 0.04 & 0.00 & 0.13 & \\
WAI & RWCS & 0.44 & 0.01 & 0.00 & 0.95 & 0.95 \\
WAI & RWCQ & 0.36 & 0.01 & 0.00 & 0.93 & 0.93 \\
\hline
\end{tabular}

\section{DISCUSSION}

Since the response rates for the questionnaire and physical examination were 25.1 and $32.1 \%$, respectively, there was concern about sample representation. However, by performing stratified random sampling according to variables of grade of permanent disability and gender, we eliminated any skewed distributions of disabilities for these two factors. The numbers of respondents to the questionnaire at different grades of disabilities exceeded 30 except in three strata, (i.e., grades 1, 3, and 4) which were probably adequate to show the variance for each stratum. Moreover, in terms of severity grades of occupational permanent disability and various demographic factors, there was no significant difference between the respondents who came and who did not come in for physical examination, as summarized in Table II. Thus, although we must be conservative in the generalization of the survey results, it might not affect the validation of the newly developed method, as the frequencies for different grades of permanent disabilities were large enough.

In this study, we tried to use the subjective rating method to measure an individual's residual work capability after occupational permanent disabilities. Instead of objectively assessing the multidimensional aspects of human work capability, we tried to ask the subject to compare his current work capability with that before the injury. Although our method could not be used to directly measure a healthy worker's work capability, it did show the subjective rating score of the residual work capability of a victim after injury.

We asked victims to self-rate their residual work capability in terms of speed, quality, and overall (combination of speed and quality) work capability (in that order). This self-rating method seemed to quantify their performance effectively as shown in Tables III and IV. Measurements of residual work capability were found to have high correlation coefficients not only with the similarly subjective WAI, but also with the more objective PCA and CSA, as performed by occupational therapists. The correlation of the RWCS, RWCQ, and RWCC questions with the
WAI, PCA, and CSA was high (all $P<0.0001$ ). We believe we have demonstrated satisfactory convergent validity for this self-rating assessment method of residual work capability with WAI, PCA, and CSA. The divergent validity with CHQ-12 also corroborated the self-rating method, as the CHQ-12 only measured the emotional aspect of Chinese people. The results of model fitting in Table IV further indicated that the perceived RWCs among workers with permanent disabilities are composite indices of physical or functional capacity, the victim's employment status after the occupational injury, the status of the victim's salary as the main source of his/her family income before injury, and the CSA. All these findings were consistently found in RWCS, RWCQ, and RWCC, with the RWCC being the most qualified single indicator for perceived residual work capability (Table IV).

The test-retest reliability of RWCQ was only 0.58 , which was lower than those of RWCS and RWCC. Because $71 \%$ (Table II) of respondents were unemployed at the time of our study, it was probably somewhat difficult for them to make consistent subjective judgments regarding their residual work capability in terms of quality. Since the reliability of RWCC was 0.77 (Pearson's correlation coefficient), we concluded that RWCC obtained from the three consecutive questions was valid and reliable, and may be considered as a simple self-rating assessment method for evaluating an injured worker's residual work capability.

WAI also had a high correlation with the more objective PCA and CSA (Table III), which indicated that WAI is a good indicator related to both physical and cognitive capability among Taiwanese people. Moreover, the model fittings shown in Table IV demonstrated that RWCC is explained by WAI with up to $52 \%$ of variance. Whether the victim's salary was the main source of income for his/her family before the injury was also a major predictor of an increase in his/her perception of RWCs. It seems that family demands or requirements improved a victim's subjective self-rating of residual work capability or facilitated such an improvement. However, the RWCC was explained by IS with only $5 \%$ of variance whether WAI is included in the 
analysis or not. Although the magnitude was relatively small in comparison with other physical attributes, one must be aware of such a possible inflating effect while using the selfrating assessment method.

Sociodemographic factors have been reported to be associated with musculoskeletal disability, including increasing age, single status, fewer years of schooling, lower income, and not being employed [Badley and Ibanez, 1994]. The age factor has also been found to especially influence work capability [Shephard, 1987]. However, in our study, we only recruited subjects with disabilities and asked them to compare conditions before and after injury, which may contribute to the null effect of these factors in the predictive models in Table IV.

\section{ACKNOWLEDGMENTS}

The authors thank the Bureau of Labor Insurance of Taiwan for kindly offering their computer files on workmen's compensation for permanent disability from occupational injury from 1/90 through 12/92.

\section{REFERENCES}

Abdel-Moty E, Fishbain DA. 1993. Functional capacity and residual functional capacity and their utility in measuring work capacity. The Clin J Pain 9:168-173.

American National Standards Institute. 1967. Method of recording and measuring work injury experience. New York: American National Standards Institute, American National Standard Z16.1.

Badley EM, Ibanez D. 1994. Socioeconomic risk factors and musculoskeletal disability. J Rheumatol 21:515-522.

Bureau of Labor Insurance for Taiwan-Fukein Area, Republic of China: Statistical data for Taiwan-Fukien labor insurance. 1984-1994.

Chang YC, Wang JD. 1995. Cumulative injury rate and potential workdays and salary lost. Scand J Work Environ Health 21:494-503.

Cheng TA, Williams P. 1986. The design and development of a screening questionnaire (CHQ) for use in community studies of mental disorders in Taiwan. Psychol Med 16:415-422.

Cheng TA, Wu JT, Chong MY, Williams P. 1990. Internal consistency and factor structure of the Chinese health questionnaire. Acta Psychiatr Scand 82:304-308.

Chong MY, Wilkinson G. 1989. Validation of 30- and 12-item versions of the Chinese Health Questionnaire (CHQ) in patients admitted for general health screening. Psycholo Med 19:495-505.
Gojer JE, Johanson U. 1991. Human resource costing and accounting. Stockholm, Sweden: Joint Industrial Safety Council, p 17-33.

Goldberg DP. 1972. The detection of psychiatric illness by questionnaire. Oxford University Press, London.

Gulick E. 1992. Model for predicting work performance among persons with multiple sclerosis. Nurs Res 41:266-272.

Laman H, Lankhorst GJ. 1994. Subjective weighting of disability: an approach to quality of life assessment in rehabilitation. Disabil Rehab 16(4): 198-204

Lechner DE, Jackson JR, Roth DL, Straaton KV. 1994. Reliability and validity of a newly developed test of physical work performance. J Occup Med 36:997-1004.

Mathiowetz V. 1990. Grip and pinch strength measurements. In: Amundsen LR, editor. Muscle strength testing. New York: Churchill Livingston, p 163-177.

Mathiowetz V. 1993. Role of physical performance component evaluations in occupational therapy functional assessment. Am J Occup Ther 47:225-230.

Mathiowetz V, Weber K, Volland G, Kashman N. 1984. Reliability and validity of grip and pinch strength evaluations. J Hand Surg 9:222.

McCormick EJ, Jeanneret PR, Mecham RC. 1989. Position analysis questionnaire. Palo Alto, CA: Consulting Psychologists Press, Inc., p 2-10.

Parry CBW. 1973. Minnesota rate of manipulation test. In: Rehabilitation of the hand (3rd ed.) Washington DC: Butterworth Inc.

Regulation of Labor Insurance. 1992. Taipei: Bureau of Labor Insurance For Taiwan-Fukein Area, ROC, p 92-130.

SAS Institute. 1990. SAS/STAT Guide for Personal Computers, Version 6 and Technical Report P-200, SAS/STAT Software: CALIS and LOGISTIC Procedures. Cary, NC: SAS Institute.

Shephard RJ. 1987. Human rights and the older worker: changes in work capacity with age. Med Sci Sports Exercise 19:168-173.

Smith HD. 1988. Assessment and evaluation-Specific evaluation procedures. In: Hopkins HL, Smith HD, editors. Willard and Spackman's occupational therapy, 7th ed. Philadelphia: J.B. Lippincott.

Tiffin J, Asher EJ. 1948. The Purdue pegboard: norms and studies of reliability and validity. J Appl Psych 32:234-247.

Tuomi K, Ilmarinen J, Eskelinen L, Jarvinen E, Toikkanen J, Klockars M. 1991. Prevalence and incidence rates of disease and work capability in different work categories of municipal occupations. Scand J Work Environ Health 17(Suppl 1):67-74.

Tuomi K, Ilmarinen J, Jahkola A, Katajarinne L, Tulkki A. 1994. Work capability index. Occupational Health Care 19. Helsinki: Institute of Occupational Health.

Velozo CA. 1993. Work evaluations: critique of the state of the art of functional assessment of work. Amer J Occup Therapy 47:203-209. 